\title{
Production porcine et ressources génétiques locales en zone périurbaine de Cotonou et d'Abomey-Calavi au Bénin
}

\author{
A.K.I. Youssao ${ }^{1} *$ G.B. Koutinhouin ${ }^{1}$ \\ T.M. Kpodekon ${ }^{1}$ A.G. Bonou ${ }^{1}$ A. Adjakpa ${ }^{1}$ \\ C.D.G. Dotcho ${ }^{2}$ F.T.R. Atodjinou ${ }^{2}$
}

\section{Mots-clés}

Porcin - Race indigène - Conduite d'élevage - Classification - Bénin.

\section{Résumé}

Une enquête sur la production porcine et les ressources génétiques locales en zone périurbaine de Cotonou et d'Abomey-Calavi au Bénin a été réalisée de juin à août 2006. Les éleveurs étaient des artisans (44 p. 100), des agroéleveurs (20 p. 100) et des petits commerçants (12 p. 100). La plupart des éleveurs achetaient des animaux (76 p. 100) pour la constitution du troupeau. La taille moyenne des troupeaux était de 19 porcs dont 4,18 truies et 1,2 verrat en moyenne. Trois types d'élevage ont été identifiés. Dans le type 1, les propriétaires d'animaux étaient des artisans. Les animaux étaient élevés en divagation le jour et passaient la nuit dans un enclos pendant la saison sèche et étaient en liberté le jour comme la nuit pendant la saison des pluies. Dans le type 2, les animaux étaient élevés en claustration et les propriétaires étaient des commerçants ou exerçaient des métiers autres que ceux de l'agriculture, de l'élevage ou de l'artisanat. Ces propriétaires accordaient peu de soins vétérinaires aux animaux. Enfin, le type 3 d'élevage regroupait les agriculteurs et les éleveurs. Dans ce type d'élevage, les animaux bénéficiaient des soins vétérinaires et des traitements traditionnels à base de plantes médicinales. Ils étaient élevés en enclos le jour comme la nuit pendant la saison des pluies et bénéficiaient également de compléments alimentaires toute I'année. Quel qu'ait été le type d'élevage, les propositions d'amélioration souhaitées par les éleveurs ont été par ordre d'importance : la formulation des aliments, les soins préventifs et curatifs, l'élevage en claustration et le regroupement en coopérative. Les éleveurs souhaitaient prioritairement une aide (assistances technique et financière) (52 p. 100) et une extension de leur exploitation (28 p. 100).

\footnotetext{
${ }^{1}$ Ecole polytechnique d'Abomey-Calavi, département de Production et Santé animales, 01 BP 2009, Cotonou, Bénin.

${ }^{2}$ Lycée agricole Médji de Sékou, Allada, Bénin.

* Auteur pour la correspondance

Tél. : +22995285988/97912074; fax : +22921360199

E-mail : issaka.youssao@epac.uac.bj
}

\section{INTRODUCTION}

Dans les pays de la sous-région ouest-africaine en général et au Bénin en particulier, la production en viande ne satisfait pas les besoins exprimés par les consommateurs. Ce déficit est comblé par des importations qui ne cessent d'augmenter tous les ans (13). En dix ans, le volume des importations de viande est passé de $16608 \mathrm{t}$ en 1993 à 86535 t en 2003 au Bénin, soit une croissance de 50 p. 100 par an sur les dix dernières années (13). Compte tenu du niveau de la consommation actuelle et de la croissance démographique qui est de 3 p. 100 par an, les besoins en viande atteindront 143000 t en 2020 (14). Pour réduire ce déficit en protéines d'origine animale, le développement au niveau national de l'élevage 
des espèces à cycle court et prolifiques (porcs, volailles...) et des espèces non conventionnelles (lapin, aulacode...) pourra y contribuer. Dans les départements de l'Atlantique et du Littoral au sud du Bénin, l'effectif estimé du cheptel est de 11700 bovins, 33700 ovins, 66700 caprins, 10980 porcins et 461000 volailles (10). Classée troisième produit de consommation après celles du bœuf et des volailles, la viande de porc a connu un regain de consommation au sud du Bénin durant ces dernières années malgré les interdits religieux (4). Ces interdits proviennent en majorité de l'islam et dans de rares cas de cultes traditionnels, et touchent la majorité de la population du Nord Bénin, à forte dominance musulmane. La viande de porc est aujourd'hui très appréciée des populations du sud du pays et des restaurants spécialisés se multiplient dans les grands centres urbains (4). Abomey-Calavi et Cotonou, à l'instar des autres villes du sud du Bénin, s'approvisionnent le plus souvent en viandes à partir des élevages périurbains. La population totale de ces deux villes représente 972845 habitants, soit 14,4 p. 100 de la population béninoise (14). Malgré leur importance numérique, les porcs locaux qui représentent 90 p. 100 du cheptel national porcin (5) sont très peu suivis sur le plan sanitaire et zootechnique (alimentation, reproduction et génétique) dans les élevages périurbains, au détriment des porcs exotiques, des volailles, des ruminants et même des lapins et des aulacodes. Cette enquête a eu pour but de caractériser l'élevage en zone périurbaine de Cotonou et d'Abomey-Calavi et de faire des propositions pour améliorer sa productivité.

\section{MATERIEL ET METHODES}

Les enquêtes se sont déroulées du $1^{\text {er }}$ juin au 30 août 2006 dans les communes d'Abomey-Calavi et de Cotonou, respectivement dans les départements de l'Atlantique et du Littoral (figure 1).

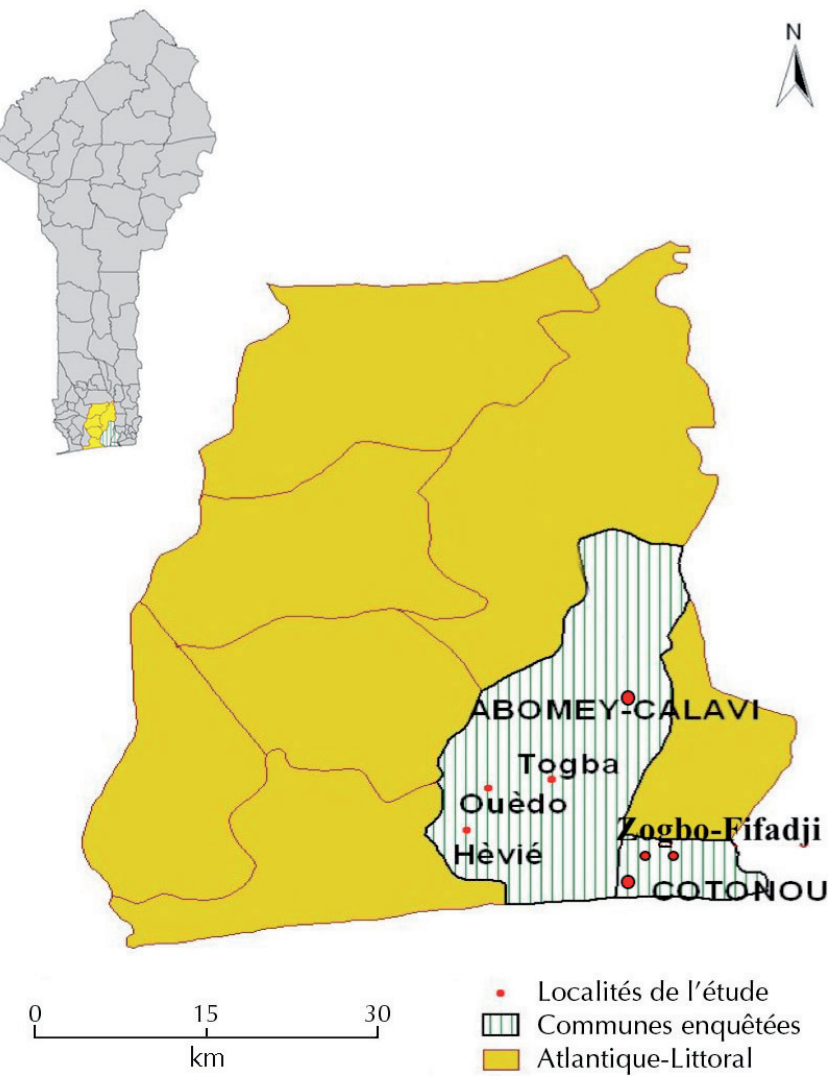

Figure 1 : zone d'étude.

\section{Matériel}

La collecte des données a été réalisée sur les porcs locaux et les éleveurs de cette race en utilisant une fiche d'enquête, une balance mécanique et une fiche d'enregistrement des données. Le porc local du Bénin, encore désigné porc Nain de l'Afrique de l'Ouest, se rencontre le long des pays côtiers de la sous-région ouest-africaine et descendrait probablement du porc européen $(9,11)$. Sur le plan phylogénétique, il est proche des sangliers italiens et turcs, et des porcs ibériques, espagnols et belges (22). Le porc local du Bénin présente des haplotypes E1 et dénote une introgression des souches européennes (comme le Large White) et asiatiques (22).

Sur le plan ethnologique, le porc local du Bénin a un museau long et cylindrique, conique et effilé à l'extrémité. Le profil est légèrement concaviligne et les oreilles sont petites, dressées et parfois rejetées en arrière. Ce sont des animaux haut sur pattes, à corps étroit ; la cuisse est plate avec un jambon peu fourni. Ils sont de petit format avec une hauteur au garrot de $40 \mathrm{~cm}$, une longueur du corps de $60 \mathrm{~cm}$ et un poids adulte de 40 à $50 \mathrm{~kg}$. La robe est généralement noire avec des extrémités blanches et parfois une ceinture blanche. Les robes blanches avec des extrémités noires ou grises se rencontrent également.

La fiche d'enquête a permis de recueillir les informations suivantes : l'identification de l'éleveur (localité, sexe, profession, etc.) ; la taille et la structure du troupeau, le statut du propriétaire (propriété exclusive ou partielle, position au sein de la famille, etc.), la conduite des animaux, l'exploitation des animaux et les contraintes liées à l'élevage.

Les données individuelles récoltées sur les truies et les porcelets ont été : le nom de la truie, sa date de naissance, son âge à la première mise bas, sa date de mise bas, son rang de mise bas, sa taille de portée à la naissance, le nombre de mort-nés à la naissance et de la naissance au sevrage par portée, les causes de mortalité, le poids à la naissance des porcelets, l'évolution du poids de la naissance au sevrage, et la généalogie. Les causes de mortalité ont été déterminées sur la base d'un diagnostic clinique effectué par le technicien d'élevage qui avait en charge la collecte des données de la présente étude.

Deux balances mécaniques de capacités maximales de 5 et $10 \mathrm{~kg}$, et de précision respective de 50 et $200 \mathrm{~g}$ ont été utilisées pour les pesées des porcelets pendant la collecte des données. Ces données de poids ont été enregistrées dans une fiche d'enregistrement.

\section{Méthodes}

Les enquêtes se sont déroulées auprès des éleveurs de porcs de race locale. Une enquête préliminaire a été d'abord réalisée à partir d'un questionnaire structuré servant de base aux discussions avec les éleveurs. Pendant la phase d'enquête proprement dite, les éleveurs ont été choisis suivant les critères d'accès à la porcherie et d'entière disposition vis-à-vis des animaux.

$\mathrm{Au}$ total, 100 éleveurs ont été enquêtés dont 68 hommes et 32 femmes. Les portées de 46 truies de races locales ont été suivies pour l'étude des paramètres zootechniques. Les performances de reproduction ont été d'abord enregistrées par truie. Les porcelets ont été ensuite pesés à la naissance (parfois jusqu'à trois jours après la mise bas) et à 14, 28, 42, 56 et 70 jours d'âge. Les pesées ont eu lieu le matin entre 7 et $9 \mathrm{~h}$. La veille des pesées, les animaux ont été mis en enclos.

\section{Analyse statistique}

Le dépouillement a été une opération au cours de laquelle les données collectées sur le terrain ont été passées en revue, codées 
et enregistrées dans une base de données conçue avec le logiciel Microsoft Excel. Après le dépouillement et l'encodage, les données ont été analysées avec le logiciel SAS (23). La procédure Proc mean a été utilisée pour la statistique descriptive. Les fréquences ont été calculées par la procédure Proc freq et les comparaisons entre les fréquences relatives ont été réalisées par le test du Chi-carré et/ou par le test bilatéral de Z. Pour chaque fréquence relative, un intervalle de confiance (IC) à 95 p. 100 a été calculé suivant la formule :

$I C P=1,96 \sqrt{\frac{[P(1-P)]}{N}}$

où $\mathrm{P}$ est la fréquence relative et $\mathrm{N}$ la taille de l'échantillon.

La procédure Proc corresp a été utilisée pour l'analyse factorielle des correspondances (AFC). Les variables prises en compte ont été : l'arrondissement de l'éleveur, l'activité principale de l'éleveur, la pratique de la divagation ou non, l'habitat, le suivi sanitaire, et la taille des troupeaux. Une classification ascendante hiérarchique sur la base des coordonnées des éleveurs sur les composantes de l'AFC les plus significatifs a été ensuite réalisée. La procédure des modèles linéaires généralisés sous SAS (23) a été utilisée pour comparer les poids à âge type des trois types d'élevage. Le test $t$ de Student a enfin été utilisé pour comparer les moyennes deux à deux.

\section{RESULTATS}

\section{Statut de l'éleveur}

Le tableau I montre les fréquences relatives sur le statut des éleveurs de porcs en zone périurbaine d'Abomey-Calavi et de Cotonou. Les éleveurs de porcs locaux dans ces communes étaient pour la plupart propriétaires exclusifs des animaux (84 p. 100). La propriété partielle a été observée chez 16 p. 100 des éleveurs. Ces élevages de type familial étaient la propriété du chef de ménage (époux) dans 80 p. 100 des cas. La part détenue par les enfants était de 8 p. 100 et le reste (8 p. 100) était la propriété des alliés. Peu de femmes étaient propriétaires d'animaux sur les 100 éleveurs enquêtés (4 p. 100). Les porcs étaient pour la plupart élevés par les artisans (44 p. 100), venaient ensuite les agroéleveurs (20 p. 100), les petits commerçants (12 p. 100) et les autres (élèves, enseignants, militaires, etc.).

\section{Constitution des troupeaux et objectifs de production}

Le tableau I présente également les fréquences en fonction du mode de constitution, des objectifs de production et l'âge des exploitations. Pour la constitution du troupeau, la plupart des éleveurs achetaient des animaux (76 p. 100) et d'autres constituaient exclusivement leur troupeau à partir des animaux qui leurs étaient confiés (16 p. 100). Des cas d'achat et de confiage (4 p. 100) ou de don et de confiage (4 p. 100) ont été aussi rencontrés. Les objectifs de production des éleveurs traditionnels de porcs locaux étaient variés : vente (96 p. 100), épargne (72 p. 100), besoins socioculturels (36 p. 100), autoconsommation (24 p. 100), autre (4 p. 100). Il est important de souligner que la plupart des éleveurs poursuivaient plusieurs objectifs en élevant ces animaux. Enfin, 52 p. 100 des éleveurs enquêtés ont démarré leur élevage il y a moins de dix ans.

\section{Structure du troupeau}

Les exploitations de porcs rencontrées au cours des enquêtes étaient de type familial et de petite taille. La taille moyenne des troupeaux était de 19 porcs dont 4,18 truies et 1,2 verrat en moyenne. Les effectifs moyens des truies vides, gravides, allaitantes, ainsi que ceux des porcelets sevrés et non sevrés sont présentés au tableau II. Les coefficients de variation étaient très élevés, ce qui témoignait de la grande variabilité de la taille des troupeaux. L'effectif des animaux a été en moyenne de 14,87 \pm 9,26 porcs par troupeau chez les femmes contre 20,94 \pm 14,26 porcs chez les hommes. Les éleveurs qui pratiquaient la divagation avaient un effectif réduit par rapport à ceux qui ne la pratiquaient pas $(12,15 \pm$ $8,24$ vs $26,42 \pm 12,59)$.

\section{Tableau I}

Statut de l'éleveur, mode de constitution du troupeau et objectifs de production

\begin{tabular}{|c|c|c|c|}
\hline $\begin{array}{l}\text { Source } \\
\text { de variation }\end{array}$ & Variable & $\begin{array}{c}\text { Fréquence } \\
\text { relative (\%) }\end{array}$ & IC \\
\hline \multirow{2}{*}{$\begin{array}{l}\text { Propriété } \\
\text { des animaux }\end{array}$} & Propriété exclusive & $84^{\mathrm{a}}$ & 7,19 \\
\hline & Propriété partielle & $16^{\mathrm{b}}$ & 7,19 \\
\hline \multirow{4}{*}{$\begin{array}{l}\text { Propriétaire } \\
\text { dans la famille }\end{array}$} & Chef de ménage & $80^{\mathrm{a}}$ & 7,84 \\
\hline & Epouse & $4^{c}$ & 2,74 \\
\hline & Fils & $8^{b}$ & 5,32 \\
\hline & Autre & $8^{b}$ & 5,32 \\
\hline \multirow{4}{*}{$\begin{array}{l}\text { Profession } \\
\text { de l'éleveur }\end{array}$} & Agroéleveur & $20^{b}$ & 7,84 \\
\hline & Commerçant & $12^{b}$ & 6,37 \\
\hline & Artisan & $44^{\mathrm{a}}$ & 9,73 \\
\hline & Autre & $24^{\mathrm{b}}$ & 8,37 \\
\hline \multirow{4}{*}{$\begin{array}{l}\text { Mode } \\
\text { de constitution }\end{array}$} & Achat & $76^{\mathrm{a}}$ & 8,37 \\
\hline & Confiage & $16^{\mathrm{b}}$ & 7,18 \\
\hline & Achat et confiage & $4^{c}$ & 3,84 \\
\hline & Don et confiage & $4^{c}$ & 3,84 \\
\hline \multirow{5}{*}{$\begin{array}{l}\text { Objectif } \\
\text { de production }\end{array}$} & Autoconsommation & $24^{c}$ & 8,37 \\
\hline & Vente & $96^{\mathrm{a}}$ & 3,84 \\
\hline & Epargne & $72^{b}$ & 8,80 \\
\hline & Besoin socioculturel & $36^{c}$ & 9,41 \\
\hline & Autre & $4^{d}$ & 3,84 \\
\hline \multirow{2}{*}{$\begin{array}{l}\text { Année } \\
\text { de démarrage }\end{array}$} & $<10$ ans & $52^{a}$ & 9,79 \\
\hline & $>10$ ans & $48^{\mathrm{a}}$ & 9,79 \\
\hline
\end{tabular}

IC : intervalle de confiance

Les fréquences relatives d'une même colonne non suivies d'au moins une lettre commune diffèrent significativement au seuil de 5 p. 100

\section{Tableau II}

Structure des troupeaux

$\begin{array}{lcccc}\text { Variable } & \text { Moyenne } & \begin{array}{c}\text { Ecart- } \\ \text { type }\end{array} & \begin{array}{c}\text { Mini- } \\ \text { mum }\end{array} & \begin{array}{c}\text { Maxi- } \\ \text { mum }\end{array} \\ \text { Verrat } & 1,20 & 0,96 & 0 & 3 \\ \text { Truie } & 4,48 & 2,35 & 1 & 10 \\ \text { Truie vide } & 1,52 & 1,58 & 0 & 6 \\ \text { Truie gravide } & 1,52 & 1,16 & 0 & 5 \\ \text { Truie allaitante } & 1,2 & 0,97 & 0 & 3 \\ \text { Porcelet sevré } & 4,44 & 4,73 & 0 & 14 \\ \text { Porcelet non sevré } & 5,8 & 6,41 & 0 & 20 \\ \text { Effectif moyen } & 19 & 13 & 2 & 48 \\ \text { des troupeaux } & & & & \end{array}$




\section{Mode d'élevage}

Les éleveurs qui élevaient les animaux en enclos (72 p. 100) ont été significativement plus nombreux $(\mathrm{P}<0,01)$ que ceux qui élevaient les animaux en liberté ( 24 p. 100) le jour, pendant la saison des pluies. La nuit, tous les éleveurs mettaient leurs animaux en enclos. Pendant la saison sèche, le mode d'élevage était le même le jour et la nuit. Au cours de cette saison, l'élevage en liberté (64 p. 100) était le plus pratiqué $(\mathrm{P}<0,01)$ par rapport à l'élevage en claustration (12 p. 100). Le tableau III donne le détail sur le mode d'élevage des porcs. Lorsque les animaux étaient en claustration, c'était le chef de ménage qui s'occupait du suivi (alimentation, entretien, etc.) dans la plupart des cas (88 p. 100), venaient ensuite les enfants et l'épouse. Les habitats étaient pour la plupart rudimentaires et ne répondaient pas souvent aux normes zootechniques.

Lorsque les animaux étaient en divagation, 70 p. 100 des éleveurs ne donnaient pas de compléments. Toutefois, les animaux étaient systématiquement nourris par l'éleveur lorsqu'ils étaient dans l'enclos. Les aliments utilisés étaient très variés et principalement constitués de tourteau de palmiste, de résidus de cuisine, de son de blé. La reproduction n'était pas suivie et les accouplements se faisaient lors de la divagation. Dans chaque élevage il y avait en moyenne 1,20 verrat pour 4,48 truies. Cependant, 60 p. 100 des éleveurs n'avaient pas de géniteurs mâles dans leurs troupeaux.

\section{Tableau III}

Mode d'élevage et suivi des animaux

\begin{tabular}{|c|c|c|c|}
\hline $\begin{array}{l}\text { Source } \\
\text { de variation }\end{array}$ & Variable & $\begin{array}{l}\text { Fréquence } \\
\text { relative (\%) }\end{array}$ & IC \\
\hline $\begin{array}{l}\text { Saison des pluies: } \\
\text { journée }\end{array}$ & $\begin{array}{l}\text { Libre } \\
\text { Enclos } \\
\text { Libre - enclos }\end{array}$ & $\begin{array}{r}24^{\mathrm{b}} \\
72^{\mathrm{a}} \\
4^{\mathrm{c}}\end{array}$ & $\begin{array}{l}8,37 \\
8,80 \\
3,841\end{array}$ \\
\hline $\begin{array}{l}\text { Saison des pluies : } \\
\text { nuit }\end{array}$ & Enclos & 100 & 0,00 \\
\hline $\begin{array}{l}\text { Saison sèche : } \\
\text { jour et nuit }\end{array}$ & $\begin{array}{l}\text { Libre } \\
\text { Enclos } \\
\text { Libre - enclos }\end{array}$ & $\begin{array}{l}64^{\mathrm{a}} \\
12^{\mathrm{b}} \\
20^{\mathrm{b}}\end{array}$ & $\begin{array}{l}9,41 \\
6,37 \\
7,84\end{array}$ \\
\hline $\begin{array}{l}\text { Suivi de l'élevage } \\
\text { en enclos }\end{array}$ & $\begin{array}{l}\text { Chef de ménage } \\
\text { Epouse } \\
\text { Fils } \\
\text { Autre }\end{array}$ & $\begin{array}{r}88^{a} \\
24^{b} \\
32^{b} \\
8^{c}\end{array}$ & $\begin{array}{l}6,369 \\
8,371 \\
9,143 \\
5,317\end{array}$ \\
\hline $\begin{array}{l}\text { Maladie ou } \\
\text { signe clinique }\end{array}$ & $\begin{array}{l}\text { Aucun } \\
\text { Amaigrissement } \\
\text { Diarrhées } \\
\text { Gale } \\
\text { Suspicion de peste } \\
\text { porcine africaine } \\
\text { Toux }\end{array}$ & $\begin{array}{r}12^{\mathrm{bc}} \\
36^{\mathrm{a}} \\
20^{\mathrm{b}} \\
8^{\mathrm{c}} \\
20^{\mathrm{b}} \\
4^{\mathrm{d}}\end{array}$ & $\begin{array}{l}6,37 \\
9,41 \\
7,84 \\
5,32 \\
7,84 \\
\\
3,84\end{array}$ \\
\hline Soin & $\begin{array}{l}\text { Traditionnel } \\
\text { Vétérinaire } \\
\text { Aucun }\end{array}$ & $\begin{array}{r}4^{\mathrm{a}} \\
24^{\mathrm{b}} \\
72^{\mathrm{c}}\end{array}$ & $\begin{array}{l}3,84 \\
8,37 \\
8,80\end{array}$ \\
\hline
\end{tabular}

IC : intervalle de confiance

Les fréquences relatives d'une même colonne non suivies d'au moins une lettre commune diffèrent significativement au seuil de 5 p. 100

\section{Suivi sanitaire et causes de mortalité}

La majorité des éleveurs (72 p. 100) n'accordaient aucun soin aux animaux. Parmi les 28 p. 100 des éleveurs qui s'occupaient du suivi sanitaire, 24 p. 100 utilisaient les produits vétérinaires et 4 p. 100 la pharmacopée traditionnelle (tableau III). Parmi les signes cliniques ou les maladies qui étaient à l'origine de la mort des animaux, l'amaigrissement venait en tête (36 p. 100), ensuite les diarrhées (20 p. 100), une suspicion de peste porcine africaine (20 p. 100), enfin, la gale et la toux (tableau III). D'autres cas de mortalité sont apparus sans que l'éleveur n'ait observé de signes cliniques ou de maladie.

\section{Exploitation des animaux}

Le tableau IV présente diverses fréquences sur l'exploitation des animaux dans la périphérie d'Abomey-Calavi et de Cotonou. Les jeunes mâles et les vieilles femelles étaient les plus vendus avec des fréquences relatives respectives de 48 et 44 p. 100. Les causes de sorties de ces animaux ont été : la vente (92 p. 100), les usages

\section{Tableau IV}

Exploitation des animaux et perspectives d'amélioration de la productivité des animaux

\begin{tabular}{|c|c|c|c|}
\hline $\begin{array}{l}\text { Source } \\
\text { de variation }\end{array}$ & Variable & $\begin{array}{l}\text { Fréquence } \\
\text { relative (\%) }\end{array}$ & IC \\
\hline \multirow[t]{5}{*}{ Animal sorti } & Verrat & $32^{\mathrm{c}}$ & 9,14 \\
\hline & Jeune mâle & $48^{b}$ & 9,79 \\
\hline & Vielle femelle & $44 b^{c}$ & 9,73 \\
\hline & Autre & $64^{\mathrm{a}}$ & 9,41 \\
\hline & Autoconsommation & $12^{\mathrm{d}}$ & 6,37 \\
\hline \multirow[t]{6}{*}{ Cause de sortie } & Don & $12^{d}$ & 6,37 \\
\hline & Fête & $76^{\mathrm{b}}$ & 8,37 \\
\hline & Mariage & $48^{\mathrm{c}}$ & 9,79 \\
\hline & Vente & $92^{\mathrm{a}}$ & 5,32 \\
\hline & Cérémonie & $48^{\mathrm{c}}$ & 9,79 \\
\hline & Autre & $4^{e}$ & 3,84 \\
\hline \multirow[t]{5}{*}{ Difficulté } & Vol & $52^{\mathrm{b}}$ & 9,79 \\
\hline & Maladie & $92^{\mathrm{a}}$ & 5,32 \\
\hline & Habitat & $64^{b}$ & 9,41 \\
\hline & Alimentation & $60^{b}$ & 9,60 \\
\hline & Autre & $4^{c}$ & 3,84 \\
\hline \multirow{3}{*}{$\begin{array}{l}\text { Solution } \\
\text { envisagée }\end{array}$} & Oui & $8^{b}$ & 5,32 \\
\hline & Non & $80^{\mathrm{a}}$ & 7,84 \\
\hline & Autre & $12^{b}$ & 6,37 \\
\hline \multirow{4}{*}{$\begin{array}{l}\text { Proposition } \\
\text { d'amélioration }\end{array}$} & Elevage en claustration & $68^{\mathrm{b}}$ & 9,14 \\
\hline & Alimentation rationnelle & $88^{\mathrm{a}}$ & 6,37 \\
\hline & Soin préventif et curatif & $88^{\mathrm{a}}$ & 6,37 \\
\hline & $\begin{array}{l}\text { Regroupement } \\
\text { en coopérative }\end{array}$ & $30^{c}$ & 8,98 \\
\hline \multirow[t]{3}{*}{ Souhait } & Extension & $28^{\mathrm{b}}$ & 8,80 \\
\hline & Aide & $52^{\mathrm{a}}$ & 9,79 \\
\hline & Financement & $4^{c}$ & 3,84 \\
\hline
\end{tabular}

IC : intervalle de confiance

Les fréquences relatives d'une même colonne non suivies d'au moins une lettre commune diffèrent significativement au seuil de 5 p. 100 
au cours des fêtes (76 p. 100), les mariages (48 p. 100) et les cérémonies (48 p. 100). Les dons et l'autoconsommation venaient en dernière position avec 12 p. 100 pour chacun d'eux.

\section{Difficultés rencontrées et solutions envisagées}

Le tableau IV présente également les difficultés rencontrées par les éleveurs de porcs locaux ainsi que les solutions envisagées. Les difficultés concernant l'élevage des porcs locaux ont été les pathologies (92 p. 100), suivies de l'alimentation et de l'habitat (64 p. 100) et enfin le vol (52 p. 100). Pour pallier ces difficultés, 80 p. 100 des éleveurs avaient peu de solutions. D'autres proposaient l'amélioration de l'habitat, et des appuis techniques et financiers pour l'entretien des animaux en claustration.

Les propositions d'amélioration souhaitées par les éleveurs ont été par ordre d'importance : l'alimentation, les soins préventifs et curatifs, l'élevage en claustration et le regroupement en coopérative (tableau IV). Les éleveurs souhaitaient prioritairement une assistance technique (52 p. 100) et une extension de leur exploitation (28 p. 100). Le financement de l'élevage venait en dernière position (4 p. 100).

\section{Perspectives et motivations des éleveurs de porcs}

En cas de maladies graves, comme la peste porcine africaine, 64 p. 100 des éleveurs refuseraient l'abattage de leurs animaux sans indemnisation et 20 p. 100 l'accepteraient. Toutefois, 16 p. 100 étaient indécis. La majorité des éleveurs de porcs (80 p. 100) envisageaient d'abandonner cet élevage contre 8 p. 100 qui souhaitaient poursuivre malgré les difficultés $(\mathrm{P}<0,01)$; 12 p. 100 étaient indécis.

\section{Productivité numérique}

Sur 46 portées correspondant à 264 porcelets, la taille moyenne de la portée a été de 5,74 $\pm 2,06$ et celle des animaux nés vivants de 4,65 $\pm 1,91$. Le taux de mort-nés a été de $18,94 \pm 4,73$ p. 100 et celui de la mortalité ente naissance et sevrage de 21,75 \pm 5,5 p. 100. Le nombre de porcelets morts dans la première semaine de mise bas a été en moyenne de 1,09 $\pm 0,91$.

\section{Typologie des élevages}

Le tableau $\mathrm{V}$ présente les valeurs propres et les proportions d'informations concentrées sur les axes. Avec les trois premiers axes, la quasi-totalité des informations de départ ont été contrôlées, garantissant une très bonne précision d'interprétation. Ces axes ont été retenus pour l'interprétation des résultats de l'analyse factorielle des correspondances $\left(\chi^{2}=72,93\right)$. En ce qui concerne la contribution

\section{Tableau V}

Valeurs propres et proportions d'informations concentrées sur les axes

\begin{tabular}{ccccc}
$\begin{array}{c}\text { Valeur } \\
\text { singulière }\end{array}$ & $\begin{array}{c}\text { Inertie } \\
\text { principale }\end{array}$ & $\chi^{\mathbf{2}}$ & $\%$ & $\%$ cumulé \\
\hline 0,2813 & 0,0791 & 50,49 & 69,23 & 69,23 \\
0,1543 & 0,0238 & 15,20 & 20,84 & 90,06 \\
0,1066 & 0,0114 & 7,24 & 9,94 & 100 \\
\hline Total & 0,1143 & 72,93 & 100,01 & - \\
\hline
\end{tabular}

absolue des différentes modalités sur chacun des axes, Fifadji a été bien représenté sur le premier (98,3 p. 100) et dans une moindre mesure sur le troisième (21,5 p. 100). Hèvié, Ouèdo, Zogbo et Togba ont été bien représentés sur l'axe 2 avec des contributions respectives de 91,4,70,5, 78,8 et 61,1 p. 100. Les contributions d'Ouèdo, de Zogbo et de Togba sur l'axe 1 ont été faibles avec des proportions respectives de 29,1,21,2 et 31,1 p. 100.

Le premier axe factoriel a opposé les animaux élevés en divagation qui passaient la nuit dans un enclos pendant la saison sèche et en liberté le jour pendant la saison des pluies, aux animaux non divagant élevés dans un enclos le jour comme la nuit pendant la saison pluvieuse. Les propriétaires d'animaux dont l'activité principale était le commerce ont également été représentés sur cet axe. Le second axe a différencié les élevages selon l'activité principale des propriétaires d'animaux (éleveurs, agriculteurs et les métiers autres que les commerçants et les artisans), d'une part, et soigner ou ne pas soigner les animaux avec des produits traditionnels ou vétérinaires, d'autre part. Enfin, les artisans ont été très bien représentés sur le troisième axe factoriel. Une classification ascendante hiérarchique réalisée sur les coordonnées de ces axes a permis de regrouper les élevages en trois catégories. La figure 2 présente la typologie des élevages sur le plan factoriel représenté par les deux premiers axes.

Quel qu'ait été le type d'élevage, pendant la saison sèche, les animaux étaient en divagation. Dans le type 1, les propriétaires d'animaux étaient des artisans. Les animaux étaient élevés en divagation et passaient la nuit dans un enclos pendant la saison sèche et étaient en liberté le jour comme la nuit pendant la saison des pluies. Ce type d'élevage a été rencontré à Fifadji, à la périphérie de Cotonou. La taille des élevages était de 23,8 \pm 11,1 porcs.

Dans le type 2, les animaux étaient élevés en claustration et les propriétaires étaient des commerçants ou ils exerçaient des métiers autres que ceux de l'agriculture, de l'élevage ou de l'artisanat. Ces propriétaires accordaient peu de soins vétérinaires aux animaux. Ce type d'élevage était plus fréquent à Zogbo, à la périphérie de Cotonou. La taille des élevages était de 23,3 \pm 8,2 porcs à Zogbo.

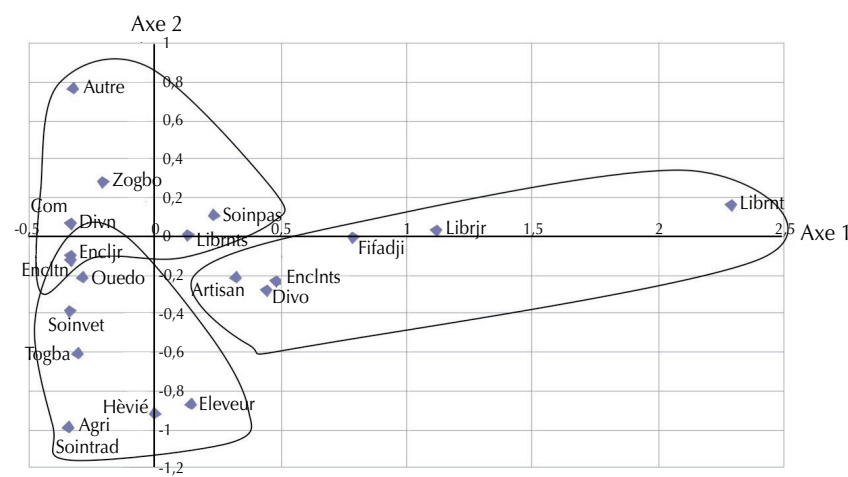

Figure 2 : typologie des élevages porcins sur le plan factoriel représentée par les deux premiers axes. Com : commerçant Agri : agriculteur ; Autre : propriétaires d'animaux autres que les agriculteurs, les éleveurs et les artisans ; Divn : animaux non divagants ; Divo : animaux en divagation ; Librnt : animaux en liberté la nuit pendant la saison des pluies ; Librir : animaux en liberté le jour pendant la saison des pluies; Librnts : animaux en liberté la nuit pendant la saison sèche; Encljr : animaux en enclos le jour pendant la saison des pluies; Enclnt : animaux en enclos la nuit pendant la saison des pluies ; Enclnts : animaux en enclos la nuit pendant la saison sèche; Soinvet : application des soins vétérinaires ; Sointrad : application des soins traditionnel. 
Enfin, dans le type 3, les propriétaires étaient des agriculteurs et des éleveurs. Les animaux bénéficiaient des soins vétérinaires et des traitements traditionnels à base de plantes médicinales. Ils étaient élevés en enclos le jour comme la nuit pendant la saison pluvieuse et bénéficiaient également de compléments toute l'année. Ce type 3 a été rencontré à Togba, Ouèdo et Hèvié, localisés à la périphérie d'Abomey-Calavi. La taille des élevages était de 17,07 \pm 5,2 porcs.

\section{Performances pondérales}

Pendant les quarante-deux premiers jours après la naissance, les porcelets des trois types d'élevage ont eu des poids identiques $(\mathrm{P}>0,05)$. A 56 jours, les animaux des élevages de type 3 ont eu des poids plus élevés $(\mathrm{P}<0,05)$ que ceux des élevages divagants de type 1 . A 70 jours, les porcelets des élevages de type 3 ont été plus lourds que ceux des élevages de type $2(\mathrm{P}<0,05)$ et ceux des élevages de type 1 ont eu les poids les plus faibles $(\mathrm{P}<0,01)$. Le tableau VI présente, par type d'élevage, l'évolution du poids des porcelets de la naissance au sevrage.

\section{DISCUSSION}

\section{Caractéristiques des élevages de porcs locaux}

A l'issue de cette étude, il est ressorti qu'il n'y avait aucun éleveur professionnel de porcs locaux car la plupart des éleveurs n'étaient ni agriculteurs, ni éleveurs. Au Tchad, les éleveurs de porcs appartiennent à toutes les couches socioprofessionnelles : agriculteurs, agroéleveurs, pêcheurs, salariés, artisans, retraités civils et militaires, élèves et étudiants, etc. (17). L'élevage de porcs constitue une alternative de diversification des revenus pour les familles, justifiant la diversité des objectifs de production, la destination des animaux et la taille des troupeaux. Au sud du Bénin, le nombre d'animaux par élevage varie suivant les régions de quatre à 18 porcs par élevage, selon Nonfon et coll. (19). Ces effectifs sont inférieurs à ceux obtenus dans la présente étude. La taille des troupeaux dans la zone d'étude était supérieure à celle des élevages traditionnels du bassin arachidier sénégalais où 86 p. 100 des éleveurs détiennent moins de quatre porcs (8). Au Tchad, en zone rurale, les effectifs varient souvent entre trois et 18 porcs, mais des exploitations de plus de 30 animaux ont été observées (17). Dans les zones urbaines et périurbaines du Tchad, les effectifs sont plus élevés et se situent entre 13 et 25 animaux (17).

Selon Devendra et Fuller (9), les aliments sont distribués une à trois fois par jour en enclos, soit sous forme de matières premières

ES : erreur standard

${ }^{\dagger}$ Poids pris dans les $72 \mathrm{~h}$ qui ont suivi la mise bas

$* \mathrm{P}<0,05 ; * * \mathrm{P}<0,01 ; \mathrm{NS}: \mathrm{P}>0,05$ simples (dans la plupart des cas) soit sous forme d'aliments composés. Peu d'éleveurs perçoivent la nécessité d'un aliment composé. Dans le cas de l'usage d'aliments simples, les éleveurs alternent périodiquement un aliment avec un autre et complémentent avec du fourrage vert. Dans le cas d'utilisation d'aliments composés, les différentes combinaisons ne répondent à aucune norme précise et se font au hasard selon les disponibilités alimentaires (2). Selon Ayssiwede (6), l'alimentation ad libitum devrait être mise en place du stade porcelet (dès la deuxième semaine) jusqu'à la phase de croissance (18 à $24 \mathrm{~kg}$ ), suivie d'un rationnement à partir de la phase d'engraissement-finition (25 à $40 \mathrm{~kg}$ ) pour les races locales élevées en claustration et nourries avec un aliment composé équilibré (6). Un aliment composé équilibré améliorerait la productivité pondérale du porc local (26) ainsi que la qualité organoleptique et nutritionnelle de sa viande (27).

Au Bénin, la majorité des porcs sont élevés traditionnellement et la conduite des troupeaux repose sur l'élevage en divagation (12). Mais les conflits avec les propriétaires de cultures saccagées par les animaux, la difficulté de maîtrise de l'animal, les vols et prédations, l'impact des maladies sur la santé publique, la propagation facile et rapide des maladies lors de la divagation, les recommandations et répressions administratives sanitaires sont les principaux facteurs qui obligent aujourd'hui la majorité des éleveurs à pratiquer une claustration permanente ou saisonnière. C'est la raison pour laquelle, dans les zones périurbaines d'Abomey-Calavi essentiellement agricoles, la plupart des élevages sont de type 3. Dans les villages de la zone soudanienne, la conduite des porcs est réglée par le calendrier des activités agricoles (17). Les porcs de race locale du Bénin disposent alors d'un habitat, même ceux élevés en divagation auxquels l'habitat sert de lieu de couchage et d'apport de compléments alimentaires éventuels. En dehors de quelques élevages disposant de bâtiments en matériaux durables (murs en briques), la plupart des porcheries sont en matériaux locaux (bois, feuilles de palme, tôles de récupération). Les habitats sont avec ou sans toit, les sols sont souvent nus et rapidement transformés en bourbier pendant la saison des pluies (1). Les animaux y sont regroupés sans distinction de sexe et d'âge quel que soit le type d'élevage. Ces conditions d'élevage baissent ainsi la productivité numérique et pondérale des animaux dans la zone d'étude (1).

Selon Dovonou (12), la saillie est naturelle pour les élevages en divagation. Dans les élevages pratiquant la claustration, la saillie est soit naturelle (regroupement des animaux sans distinction de sexe), soit contrôlée par l'éleveur. Dans ce dernier cas, l'éleveur se base sur le format des femelles et/ou les manifestations de cha-

\section{Tableau VI}

Poids individuel des porcelets et taille des troupeaux par type d'élevage

\begin{tabular}{|c|c|c|c|c|c|c|c|}
\hline Variable & $\begin{array}{c}\text { Type } 1 \\
\text { Moyenne }\end{array}$ & ES & $\begin{array}{c}\text { Type } 2 \\
\text { Moyenne }\end{array}$ & ES & $\begin{array}{c}\text { Type } 3 \\
\text { Moyenne }\end{array}$ & ES & $\begin{array}{c}\text { Test de } \\
\text { signification }\end{array}$ \\
\hline Poids à la naissance ${ }^{\dagger}(\mathrm{g})$ & 841,2 & 45,5 & 797,7 & 35,6 & 755,9 & 54,6 & NS \\
\hline Poids à 14 jours (g) & 1212,6 & 35,3 & 1197,9 & 44,3 & 1150,9 & 36,8 & NS \\
\hline Poids à 28 jours (g) & 1768,4 & 64,9 & 1771,5 & 77,7 & 1659,8 & 70,5 & NS \\
\hline Poids à 42 jours (g) & 2118,5 & 44,4 & 2221,1 & 52,5 & 2244,4 & 41,1 & NS \\
\hline Poids à 56 jours (g) & $2253,3^{\mathrm{a}}$ & 35,5 & $2446,7^{\mathrm{ab}}$ & 40,4 & $2621,1^{b}$ & 43,3 & $*$ \\
\hline Poids à 70 jours (g) & $2988,8^{a}$ & 64,2 & $3429,6^{b}$ & 55,7 & $3714,8^{c}$ & 49,9 & $* *$ \\
\hline
\end{tabular}


leurs pour mettre les cochettes à la saillie. Les verrats utilisés sont alors empruntés à d'autres élevages, achetés pour la circonstance et revendus ou castrés après la saillie. Dans les conditions de saillies naturelles, celles-ci sont souvent précoces. Le sevrage est souvent naturel et tardif (deux à trois mois). Les résultats issus de la présente étude sur le mode de reproduction et le ratio verrats - truies confirment ainsi ceux de Dovonou (12).

Comme déjà mentionné, l'amaigrissement et les diarrhées, les deux principaux signes cliniques et maladies à l'origine de la mort des animaux, étaient dans la plupart des cas dus aux parasitoses gastro-intestinales. Selon une enquête réalisée auprès de 165 élevages dans les principaux départements d'élevage de porcs au Bénin (Atlantique, Mono, Ouémé, Zou), les maladies les plus évoquées par les éleveurs sont les parasitoses internes et externes (59 p. 100) avec en premier lieu la gale sarcoptique (85 p. 100), suivie de la strongylose et de l'ascaridose (65 p. 100), notamment dans les élevages traditionnels et semi-intensifs (5). Un déparasitage des animaux à intervalles réguliers pourrait permettre de réduire le taux de mortalité (5). Selon Lokossou (15), la promotion de la porciculture au Bénin ne deviendra une réalité que par la formation de l'association des éleveurs de porcs, l'évolution de la vision des fermes d'élevage de l'Etat vers les centres de démonstration et de diffusion des innovations technologiques, et l'amélioration génétique des animaux. Ces propositions ont été mises en œuvre dix ans plus tard, après l'apparition, en 1997, pour la première fois de la peste porcine africaine au Bénin (21).

\section{Productivité numérique}

Sur 46 portées, le nombre moyen de porcelets nés vivants a été de 4,74. A la ferme d'élevage de Kpinnou, la taille moyenne des porcelets nés vivants est de 6,31 (7). Cette portée est plus élevée que celle obtenue au cours de la présente enquête et serait sans doute liée au mode d'élevage : à la Ferme de Kpinnou, les conditions d'élevages (habitat, suivi sanitaire, alimentation, etc.) sont nettement meilleures que celles des élevages traditionnels. Au cours de la collecte des données, la taille de la portée n'a pas pu être enregistrée dans la mesure où les truies mettaient bas à l'insu des éleveurs et dans des endroits discrets. La plupart des éleveurs ne s'en rendaient compte que 24 à $72 \mathrm{~h}$ après. Etant donné le nombre moyen d'animaux nés vivants, il est possible de déduire que la taille de la portée obtenue dans la présente étude était relativement faible comparée à celle observée par d'Orgeval Dubouchet (11) au sud du Bénin (tableau VII). La taille de la portée varie de 6,7 à 7,8 chez les porcs locaux (24) et de 8,9 à 9,6 chez les porcs exotiques élevés en milieu tropical (11). En Guinée Bissau, la taille moyenne de la portée est de cinq porcelets chez la race locale Crioulo (16).

Le nombre de porcelets morts dans la première semaine de mise bas a été de 1,09. Au cours des enquêtes, 21,74 p. 100 des cas de mortalité ont été observés de la naissance au sevrage. Ce taux n'était que de 9,05 p. 100 à la ferme de Kpinnou (7), grâce aux conditions d'élevage. Un taux de mortalité de 29,12 p. 100 a été obtenu par Nonfon et coll. (20) lors de l'étude des paramètres de productivité du porc local obtenus au Centre de recherche et de développement du porc local de 1989 à 1994 à la faculté des Sciences agronomiques de l'Université d'Abomey-Calavi. Des taux inférieurs à celui obtenu au cours des enquêtes ont été observés par Dovonou (12) chez les porcs locaux. Bien avant l'apparition de la peste porcine africaine en 1997 au Bénin (21), aucune période spécifique n'a été associée à des surmortalités. Dans certains pays comme la Guinée Bissau, les mortalités dues aux pathologies sont également enregistrées toute l'année (16). Toutefois, les plus forts taux de mortalité sont enregistrés de juin à août et correspondent à la saison chaude (16). Le tableau VII récapitule les taux de mortalité de la naissance au sevrage des porcelets et les tailles de portées en fonction de la race et du milieu.

\section{Performances pondérales}

Le poids à la naissance obtenu dans la présente étude a été similaire à ceux obtenus par d'Orgeval Dubouchet (11), et Nonfon et coll. (20) qui ont respectivement observé 0,92 et $0,96 \mathrm{~kg} 48$ heures

\section{Tableau VII}

Performances de reproduction des porcs de race locale et de races exotiques

\begin{tabular}{|c|c|c|c|c|c|c|}
\hline Race & Lieu & $\begin{array}{l}\text { Nb. de } \\
\text { portées }\end{array}$ & $\begin{array}{l}\text { Taille de la portée } \\
\text { à la naissance }\end{array}$ & $\begin{array}{c}\text { Poids à la } \\
\text { naissance (kg) }\end{array}$ & $\begin{array}{l}\text { Mortalité au } \\
\text { sevrage (\%) }\end{array}$ & Source \\
\hline Locale & Bénin * & 46 & 5,74 & 0,81 & 21,75 & Présente étude \\
\hline Locale & Bénin (Epac) & 122 & 7,25 & 0,530 & 12,6 & Youssao et coll., 2009 \\
\hline Locale & Bénin (FSA) & - & 7,31 & 0,92 & 19,1 & d'Orgeval Dubouchet, 1997 \\
\hline Locale & Bénin (Epac) & 64 & 7,67 & 0,54 & 38,89 & Alassane, 2007 \\
\hline Locale & Bénin (FEK) & 82 & 6,31 & 0,51 & - & Bonou, 2006 \\
\hline Locale & Nigeria & - & 6,7 & 0,91 & 15,8 & d'Orgeval Dubouchet, 1997 \\
\hline Locale & Cameroun & - & 7,8 & 1,15 & 22 & d'Orgeval Dubouchet, 1997 \\
\hline Large White & Nigeria & 289 & 8,9 & - & 21,4 & d'Orgeval Dubouchet, 1997 \\
\hline Landrace belge & Nigeria & 167 & 9,2 & - & 22,2 & d'Orgeval Dubouchet, 1997 \\
\hline Landrace belge & Cameroun & 532 & 9,6 & - & 16,6 & d'Orgeval Dubouchet, 1997 \\
\hline Large White & Cameroun & 85 & 9,3 & - & 27,9 & d'Orgeval Dubouchet, 1997 \\
\hline Large White & Sénégal & 510 & 9,3 & - & 15,9 & d'Orgeval Dubouchet, 1997 \\
\hline Croisé & Nigeria & 432 & 7,8 & - & 22,8 & d'Orgeval Dubouchet, 1997 \\
\hline $\begin{array}{l}\text { Large White } \mathrm{x} \\
\text { Landrace }\end{array}$ & Bénin (FEK) & 112 & 6,86 & 1,04 & 9,66 & Bonou, 2006 \\
\hline
\end{tabular}

* Elevage traditionnel

Epac : Ecole polytechnique d'Abomey-Calavi ; FSA : Faculté des Sciences agronomiques de l'Université d'Abomey-Calavi ; FEK : ferme d'élevage de Kpinnou 
après la mise bas. Le poids moyen des porcelets était de $0,51 \mathrm{~kg}$ à la ferme de Kpinnou (7) et de 0,54 à la ferme pilote du département de Production animale de l'Ecole polytechnique d'AbomeyCalavi (Epac) $(3,25)$. Ces poids sont beaucoup plus faibles mais plus précis car la prise de poids a été réalisée deux à trois heures après la mise bas.

Le poids au sevrage des porcelets a été plus important dans les élevages de type 3. Cela était certainement lié au mode d'élevage dans lequel les animaux étaient élevés : en claustration avec un apport de compléments alimentaires et un suivi sanitaire. Toutefois, ces conditions étaient moins satisfaisantes qu'à la ferme de Kpinnou ou qu'à celle de l'Epac où les animaux étaient nourris par un aliment complet et bénéficiaient d'un suivi sanitaire planifié. A la ferme de Kpinnou, le poids au sevrage était de $4,45 \mathrm{~kg}$, correspondant à un âge moyen de 58 jours (7). De même, à la ferme de l'Epac, le poids au sevrage était de 4,55 $\mathrm{kg}$ pour un âge au sevrage de 61,96 jours (24).

\section{- CONCLUSION}

Les enquêtes sur la production porcine et les ressources génétiques locales en zone périurbaine de Cotonou et d'Abomey-Calavi ont révélé l'inexistence d'éleveurs professionnels de porcs locaux.
L'élevage des porcs locaux était une activité secondaire des propriétaires d'animaux. Pendant la saison des pluies, la plupart des éleveurs élevaient les animaux en enclos dans la journée. En revanche, la nuit, tous les éleveurs mettaient leurs animaux en enclos. Pendant la saison sèche, le mode d'élevage restait le même le jour et la nuit. Au cours de cette saison, l'élevage en liberté était le plus pratiqué par rapport à l'élevage en claustration. Lorsque les animaux étaient en divagation, la plupart des éleveurs ne donnaient pas de compléments. Toutefois, les animaux étaient systématiquement nourris par l'éleveur lorsqu'ils étaient dans les enclos. La reproduction n'était pas suivie et plus de la moitié des éleveurs n'avaient pas de géniteur mâle dans leur troupeau. Pour le suivi sanitaire, la majorité des éleveurs n'accordaient aucun soin aux animaux. La taille de la portée était faible et un sur quatre porcelets mourait entre la naissance et le sevrage. Le poids et la taille de la portée étaient également faibles. Ces élevages ont été classés en trois types : type 1, les artisans pratiquaient l'élevage en divagation totale ; type 2, les commerçants, les instituteurs et les retraités élevaient les animaux en claustration ; type 3, les agriculteurs et les éleveurs appliquaient les soins vétérinaires, la médecine traditionnelle et l'élevage en claustration. Le mode d'élevage a influencé les performances de croissance des animaux qui ont été médiocres lorsque les animaux étaient élevés en divagation et se sont améliorées dans les types 2 et 3 en claustration.

\section{BIBLIOGRAPHIE}

1. ABOH A.B., OUEDRAOGO S., RIVERA A.M., PHAM TH H.H., MEKHTOUB K., 2001. Importance, contraintes et voies de développement des élevages urbains et périurbains dans la région SudBénin. Copenhagen, Denmark, University of Copenhagen. www.poultry. life.ku.dk (consulted 2 June 2009)

2. AGBOKOUNOU A.M., 2001. Etude des besoins énergétiques et protéiques du porc local béninois en phase de démarrage - croissance. Mém. DEA Zootechnie, Faculté universitaire des sciences agronomiques de Gembloux, Belgique, $92 \mathrm{p}$.

3. ALASSANE Y., 2007. Amélioration génétique des performances de croissance du porc local du Bénin par croisement avec le Large White. Mém. Ingénieur Travaux, Université d'Abomey-Calavi, Bénin, 52 p.

4. ATODJINOU F.T.R., DOTCHO C.D.G., 2006. Caractéristiques de l'élevage des porcs locaux dans les élevages périurbains de Cotonou et d'Abomey-Calavi. Mém. Diplôme d'études agricoles tropicales, Lycée Meidji de Sékou, Bénin, 80 p.

5. AYSSIWEDE S.B., 2004. La filière porcine au Benin : production, commercialisation, propositions d'amélioration et perspectives de développement. Dakar, Sénégal, Ecole Inter-Etats des Sciences et de médecine vétérinaire, $120 \mathrm{p}$.

6. AYSSIWEDE B.S., 2005. L'insémination artificielle porcine : une perspective pour I'amélioration de la productivité des porcs au Bénin. Mém. DES Gestion des ressources animales et végétales en milieux tropicaux, faculté de Médecine vétérinaire, Liège / FusaGx, Gembloux, Belgique, $85 \mathrm{p}$.

7. BONOU D., 2006. Etude comparative de quelques performances zootechniques des porcs de race locale et métisse issue du croisement entre Large White et Landrace. Mém. Ingénieur Travaux, Université d'Abomey-Calavi, Bénin, $72 \mathrm{p}$.

9. DEVENDRA C., FUlLER M., 1979. Pigs production in the tropics. Oxford, UK, Oxford University Press, 154 p.

10. DIRECTION DE L'ELEVAGE, 2008. Rapport annuel d'activité. Cotonou, Bénin, direction de l'Elevage, 112 p.

11. D'ORGEVAL DUBOUCHET R., 1997. Le développement de l'élevage porcin en Afrique: I'analyse des systèmes d'élevage du porc local africain au Sud Bénin. Thèse Doct. INA, Paris - Grignon, France, 273 p.

12. DOVONOU N., 2002. Performances zootechniques des races porcines au sud Bénin et perspectives d'amélioration par croisement avec le Piétrain stress négatif. Mém. DES Gestion des ressources animales et végétales en milieux tropicaux. Liège, Belgique, Université de Liège, 74 p.

13. FAO, 2008. FAOSTAT : Agriculture. http://apps.fao.org/page/collecti ons? subset=agriculture\&language=FR (consulté le 15 juin 2008)

14. INSAE, 2008. Rapport annuel d'activités. Cotonou, Bénin, Institut national de statistique et de l'analyse économique, 360 p.

15. LOKOSSOU H.R., 1986. L'industrialisation de l'élevage, base de la production porcine en République populaire du Bénin, étude du modèle Agrocap au Sénégal. Paris, France, Agence de coopération culturelle et technique, $111 \mathrm{p}$.

16. MARTINHO DE ALMEIDA A., ALFARO CARDOSO L., 2008. Animal production and genetic resources in Guinea Bissau, II, Tombali province. Trop. Anim. Health Prod., 40: 537-543.

17. MOPATE L.Y., 2002. Productivité des élevages porcins dans les départements du Logone occidental, du Mayo-Dallah et de la Kabia en zone soudanienne du Tchad. Rapport de mission. N'Djamena, Tchad, Lrvz, $15 \mathrm{p}$.

18. MOPATE LOGTENE Y., KOUSSOU M.O., 2002. L'élevage porcin, un élevage ignoré mais pourtant bien implanté dans les agro-systèmes ruraux et périurbains du Tchad. In : Actes Coll., Garoua, Cameroun, 27-31 mai 2002, 9 p.

19. NONFON W.R., DEKA E., ADEBGIDJI A., CODJO B., 1994. L'élevage du porc local dans le Sud Bénin. Rapport d'enquêtes diagnostiques sur systèmes d'élevage et filière de commercialisation. Cotonou, Bénin, Rdpl/FSA, 64 p. 
20. NONFON W.R., DEKA E., ADEGBIDJI A., CODJO B., CHRYSOSTOME C., 2000. Amélioration de la productivité du porc local au sud Bénin. Rapport technique final. Cotonou, Bénin, Université nationale du Bénin/FSA, 174 p.

21. OIE, 2001. African swine fever in Benin. Revue sci. tech. Off. int. Epizoot., 14: 123-129.

22. RAMIREZ O., TOMAS A., CLOP A., GALMANOMITOGUN O., MAKUZA S.M., CADILLO J.M., KELLY L., ARMAND SANCHEZ A., AMILLS M., 2006. Microsatellite and chromosome $Y$ sequence analysis of wild boar and autochthonous pig breeds from Asia, Europe, South America and Africa. In: Proc 30th Int. Conf. Animal Genetics, Porto Seguro, Brazil, 2006. Belo Horizonte, Brazil, CBRA, p. 32.

23. SAS, 1991. SAS/STAT, User's guide, $4^{\text {th }}$ Edn, Vers. 8. Cary, NC, USA, SAS Institute.

24. YOUSSAO A.K.I., KOUTINHOUIN G.B., KPODEKON T.M., BONOU A.G., ADJAKPA A., AHOUNOU G.S., MOUROT J., 2009. Performances zootechniques et aptitudes bouchères des porcs locaux au sud du Bénin. Bull. Anim. Health. Prod. Afr., 57: 73-87.
25. YOUSSAO A.K.I., KPODEKON T.M., KOUTINHOUIN G.B., ADJAPKA A., YACOUBOU A., AHOUNOU S., 2008. Influence de la castration des mâles sur les performances de croissance, les caractéristiques de la carcasse et les qualités de la viande du porc local du Bénin. Bull. Rech. Agr. Bénin, 61 : 17-24.

26. YOUSSAO A.K.I., MOUROT J., GBANGBOCHE A.B., ADEHAN R., AKOUTEY A., EDENAPKO A., 2004. Influence du régime alimentaire sur les performances de croissance et les caractéristiques de la carcasse du porc local au Bénin. Revue Afr. Santé Prod. Anim., 2 : 31-36.

27. YOUSSAO A.K.I., MOUROT J., GBANGBOCHE A.B., ADEHAN R., HORNICK J.-L., AKOUTEY A., EDENAPKO A., CLINQUART A. 2004. Influence du régime alimentaire sur les qualités technologiques et nutritionnelles de la viande du porc local du Bénin. Revue Afr. Santé Prod. Anim., 2 : 112-118.

Accepté le 10.11.2009

\section{Summary}

Youssao A.K.I., Koutinhouin G.B., Kpodekon T.M., Bonou A.G., Adjakpa A., Dotcho C.D.G., Atodjinou F.T.R. Pig Production and Indigenous Genetic Resources in Suburban Areas of Cotonou and Abomey-Calavi in Benin

A survey on pig production and indigenous genetic resources in suburban areas of Cotonou and Abomey-Calavi in Benin was carried from June to August 2006. The breeders were craftsmen (44\%), crop/livestock farmers $(20 \%)$ and small scale merchants $(12 \%)$. Most breeders purchased animals to build their herds (76\%). The average size of the herd consisted of 19 pigs, of which 4.18 were sows and 1.2 breeding boar on average. Three types of breeding systems were identified. In Type 1 , the animal breeders were craftsmen. The animals were left to roam and kept in the pens at night during the dry season, and they were left to roam day and night during the rainy season. In Type 2, the animals were kept in the pens; the owners were merchants or had an occupation other than those linked to farming, breeding, or craft trades. These breeders provided little veterinary care to their animals. Lastly, Type 3 consisted of farmers and breeders. In this breeding system type, the animals received veterinary care and traditional treatments with medicinal plants. They were kept in the pens day and night during the rainy season, and received a feed supplement all year round. For all the types of breeding systems, the suggestions for improvement made by the pig breeders were in order of importance: feeding, preventive and curative care, pen confinement, and cooperative creation. They especially expressed their wish for technical and financial support, (52\%) and to expand their activity (28\%).

Keywords: Swine - Land race - Livestock management Classification - Benin

\section{Sumario}

Youssao A.K.I., Koutinhouin G.B., Kpodekon T.M., Bonou A.G., Adjakpa A., Dotcho C.D.G., Atodjinou F.T.R. Producción porcina y recursos genéticas locales en la zona peri urbana de Cotonou y de Abomey-Calavi en Benin

Se realizó una encuesta sobre la producción porcina y los recursos genéticos locales en la zona peri urbana de Cotonou y de Abomey-Calavi en Benin, entre junio y agosto 2006. Los criadores eran artesanos (44\%), productores agrícolas $(20 \%)$ y pequeños comerciantes $(12 \%)$. La mayoría de los criadores compraron animales (76\%) para formar un hato. El tamaño medio de los hatos fue de 19 cerdos, de los cuales 4,18 hembras y 1,2 verracos en promedio. Se identificaron tres tipos de criaderos. En el tipo 1, los propietarios de los animales eran artesanos. Los animales eran criados en deambulación y pasaban la noche en un encierro durante la estación seca y en libertad durante el día como durante la noche durante la estación de lluvias. En el tipo 2, los animales eran criados en confinamiento y los propietarios de los animales eran comerciantes o ejercían oficios otros que los de la agricultura, la cría o la artesanía. Estos propietarios ofrecían pocos cuidados veterinarios a los animales. Finalmente, el tipo 3 de cría incluía a los agricultores y a los criadores. En este tipo de cría, los animales beneficiaban de cuidados veterinarios y los tratamientos tradicionales a base de plantas medicinales. Eran criados en corrales tanto durante el día como la noche durante la estación de lluvias y beneficiaban igualmente de complementos alimenticios todo el año. Cualquiera que haya sido el tipo de cría, las proposiciones de mejoría deseadas por los criadores fueron, por orden de importancia: la formulación de los alimentos, los cuidados preventivos y curativos, la cría en confinamiento y la reunión en cooperativa. Los criadores desearon prioritariamente una ayuda (asistencias técnica y financiera) (52\%) y una extensión de las explotaciones $(28 \%)$.

Palabras clave: Cerdo - Raza indígena - Manejo del ganado Clasificación - Benin. 